\title{
A Blind Search for Prompt Gamma-ray Counterparts of Fast Radio Bursts with Fermi-LAT Data
}

\author{
Shotaro Yamasaki`, Tomonori Totani, and Norita Kawanaka \\ Department of Astronomy, School of Science, The University of Tokyo, Hongo, Bunkyo-ku, Tokyo 113-0033
}

7 June 2019

\begin{abstract}
Fast Radio Bursts (FRBs) are a mysterious flash phenomenon detected in radio wavelengths with a duration of only a few milliseconds, and they may also have prompt gamma-ray flashes. Here we carry out a blind search for msec-duration gamma-ray flashes using the 7-year Fermi Large Area Telescope (Fermi-LAT) all-sky gamma-ray data. About 100 flash candidates are detected, but after removing those associated with bright steady point sources, we find no flash events at high Galactic latitude region $\left(|b|>20^{\circ}\right)$. Events at lower latitude regions are consistent with statistical flukes originating from the diffuse gamma-ray background. From these results, we place an upper limit on the $\mathrm{GeV}$ gamma-ray to radio flux ratio of FRBs as $\xi \equiv\left(\nu L_{\nu}\right)_{\gamma} /\left(\nu L_{\nu}\right)_{\text {radio }} \lesssim(4.2-12) \times 10^{7}$, depending on the assumed FRB rate evolution index $\beta=0-4$ [cosmic FRB rate $\Phi_{\mathrm{FRB}} \propto(1+z)^{\beta}$ ]. This limit is comparable with the largest value found for pulsars, though $\xi$ of pulsars is distributed in a wide range. We also compare this limit with the spectral energy distribution of the 2004 giant flare of the magnetar SGR $1806-20$.
\end{abstract}

Key words: stars: neutron, magnetars - pulsars: general - gamma-rays: general - methods: data analysis

\section{INTRODUCTION}

Fast radio bursts (FRBs) are a population of radio transients with durations of only about $1 \mathrm{msec}$. Since the discovery of the socalled Lorimer Burst (Lorimer et al. 2007), 17 FRBs have been reported by radio transient surveys to date ( 15 by Parkes radio telescope, Lorimer et al. 2007; Keane et al. 2012; Thornton et al. 2013; Burke-Spolaor \& Bannister 2014; Petroff et al. 2015; Ravi et al. 2015; Champion et al. 2015; Keane et al. 2016; 1 by Arecibo, Spitler et al. 2014; Spitler et al. 2016; Scholz et al. 2016; and 1 by Green Bank Telescope, Masui et al. 2015). Their dispersion measures (DMs) are much larger than those expected for objects in our Galaxy, and a cosmological distance scale of $z \sim$ $0.5-1$ is inferred if the dominant contribution to DMs is from electrons in intergalactic meduim. A recent study of FRB150418 has identified its radio afterglow associated with an elliptical galaxy, whose redshift $(z=0.492)$ is consistent with that expected by its DM (Keane et al. 2016). However, no counterparts in other wavelengths have been detected. There are various progenitor models proposed for FRBs; some of them are related to young stellar populations, while others to old. The former includes giant flares from soft gamma-ray repeaters (SGRs; Popov \& Postnov 2010; Thornton et al. 2013; Lyubarsky 2014; Kulkarni et al. 2014), collapsing supermassive neutron stars ("blitzar" model; Falcke \& Rezzolla 2014), and giant radio pulses

^ E-mail: yamasaki@astron.s.u-tokyo.ac.jp from pulsars (Connor et al. 2015; Cordes \& Wasserman 2015). The latter includes binary neutron star (or black hole) mergers (Totani 2013; Mingarelli et al. 2015), and binary white dwarf mergers (Kashiyama et al. 2013).

Whatever the progentor is, it is reasonable to expect corresponding msec-duration radiations of FRBs in other wavelengths. Here we search for gamma-ray counterparts of FRBs using the 7year Fermi Large Area Telescope (Fermi-LAT) data. Radio telescopes finding FRBs at cosmological distances have narrower fieldof-views ( $\sim 15$ arcmin beam size for Parkes) than Fermi-LAT, and hence we expect that Fermi-LAT detects FRBs at smaller distances than those found in radio bands. Therefore, rather than examining the Fermi-LAT data at known FRB locations, we perform a blind search of msec-duration gamma-ray flashes (MGFs), without prior information about FRBs in radio bands. Then we can derive more stringent constraints on the $\mathrm{GeV}$ gamma-ray to radio flux ratio of FRBs, than that from upper limits on gamma-ray flux set by FermiLAT for individual radio-detected FRBs.

The outline of this paper is as follows. In section 2, we describe the details of the gamma-ray data set and our search method. We then present the resuls of the MGF search in section 3. Constraints on the gamma-ray to radio flux ratio of FRBs will be derived in section 4 , followed by discussion for implications. Throughout this paper, we assume a flat-universe $\Lambda$ CDM model with $H_{0}=67.8 \mathrm{~km} \mathrm{~s}^{-1} \mathrm{Mpc}^{-1}, \Omega_{M}=0.308, \Omega_{\Lambda}=0.692$ (Planck Collaboration et al. 2015) 


\section{DATA AND SEARCH METHOD}

The Fermi-LAT is a pair-conversion gamma-ray telescope designed to cover the energy band from $20 \mathrm{MeV}$ to greater than $300 \mathrm{GeV}$. In this work we use the Pass 7 Reprocessed weekly data publicly available at the Fermi mission website ${ }^{1}$. The analysis spans the time period of 6.8 years from July 31, 2008 (UTC) to June 18, 2015 (UTC), corresponding to the mission elapsed time (MET) from $239557417 \mathrm{~s}$ to $455059763 \mathrm{~s}$ and the Fermi mission week from 1 to 368 . We use P7SOURCE class photons with reconstructed energies from 1 to $100 \mathrm{GeV}$. We used the Fermi Science Tools version v9r32p5 package ${ }^{2}$ and the P7REP instrument response functions (IRFs) in our analysis. In accordance with the data analysis procedure outlined in Fermi mission website, we firstly selected $1 \mathrm{GeV}<E<100 \mathrm{GeV}$ photons of "P7SOURCE" class. There is another "Transient class", which is for gamma-ray events with looser cuts than P7SOURCE and hence includes more background particle contamination. Though this class is useful for bright transients during which background contamination is negligible, we use P7SOURCE because we will search faint (i.e., small number statistics) transient events, to which background contamination is crucial. We also set the Earth relative zenith angle cut of $100^{\circ}$ to reduce the bright earth-limb gamma-rays. Finally we select gammarays only in the good time intervals (GTIs) when the data quality is good, by excluding the bad time intervals (BTIs) due to the spacecraft events. The total amount of GTIs is $79 \%$ of the MET.

In order to search for MGF candidates, we apply a search algorithm as follows. First we define the search time window $\Delta t$, and four values of $\Delta t=1,2,5$, and $10 \mathrm{msec}$ are tried here. We consider a reference gamma-ray event, and other gamma-ray events are searched within $2^{\circ}$ radius from the reference in the time interval of $\Delta t$ starting from the reference event. The radius is determined considering the per-photon angular resolution, $\sim 0.8^{\circ}(68 \%$ containment of the point-spread function) at $1 \mathrm{GeV}$ (Atwood et al. 2009; Acero et al. 2015). The number of gamma-rays in this time window and the circle, including the first reference photon itself, is denoted as $N_{\mathrm{ph}}$. Then we repeat this procedure for all the gammaray events of the Fermi data set that we use. It is possible that a gamma-ray event is included in different MGFs as defined above, but we present all MGF candidates as different events in the following if they are different as a combination of photons. The detector deadtime per event of Fermi-LAT is $<100 \mu$ s, and it is negligible in our analysis.

\section{RESULT}

\subsection{MGFs Associated with Known Gamma-ray Sources}

The result of our blind search for MGFs is shown in Table 1 (see columns of "No cut"). For $N_{\mathrm{ph}}=2$, there are 17, 33, 68 and 133 MGF candidates for $\Delta t=1,2,5$ and $10 \mathrm{~ms}$, respectively, as shown in the Table. Only one MGF event was found for $N_{\mathrm{ph}}=3$ and $\Delta t=5$ or $10 \mathrm{~ms}$, and there were no events of $N_{\mathrm{ph}} \geqslant 4$ for any value of $\Delta t$. We examined the $N_{\mathrm{ph}}=3$ event, and found that it is associated with GRB 090510 (Ackermann et al. 2010), which shows photon clusterings within a time-scale of $<10 \mathrm{~ms}$. The total duration of detected LAT gamma-rays for GRB 090510 is $0.1 \mathrm{~s}$, which is much longer than FRBs. Therefore we remove this event

1 http://fermi.gsfc.nasa.gov/ssc/data/access/

2 http://fermi.gsfc.nasa.gov/ssc/data/analysis/software/v9r32p5.html

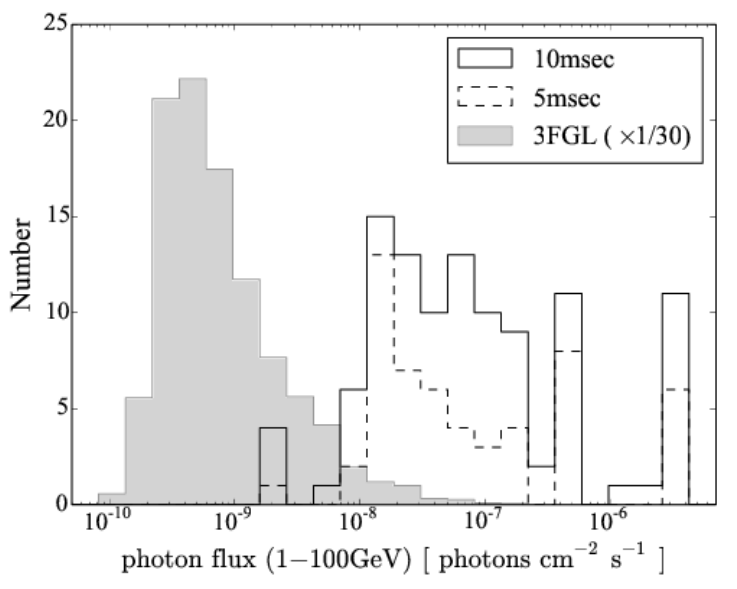

Figure 1. The photon flux $(1 \mathrm{GeV}<E<100 \mathrm{GeV})$ distribution of the nearest point source to each MGF candidate with $N_{\mathrm{ph}}=2$ and $\Delta t=5$ or $10 \mathrm{msec}$, in comparison with that of all sources in the 3FGL catalog. The histogram for the $3 F G L$ sources is multiplied by a factor of $1 / 30$ for the presentation purpose.

from our sample in the following, and the results after this " 1 st cut" are shown in Table 1 . Only $N_{\mathrm{ph}}=2$ events remain.

In order to examine the possibility that the detected $N_{\mathrm{ph}}=2$ events are caused by bright point sources, we examined the nearest point source from each MGF event. We use the Fermi Large Area Telescope Third Source Catalog (3FGL; Acero et al. 2015) which consists of $\sim 3000$ gamma-ray sources. Figure 1 presents the photon flux distribution of the nearest point sources, in comparison with that of all the 3FGL catalog point sources. The 3FGL sources nearest to the MGF candidates have obviously much brighter fluxes compared with the general 3FGL sources, indicating that most MGF candidates are caused by bright point sources. Figure 2 is the histogram of angular distance from the MGF candidates to the nearest 3FGL sources. The angular distribution is similar to the FermiLAT point spread functions (PSF; Atwood et al. 2009; Acero et al. 2015), and much smaller than the distribution expected for the case that the MGF candidates are randomly distributed in the sky, again indicating that these are caused by bright point sources. The classes of the nearest sources include a variety of populations for which msec scale variability is physically unlikely (e.g. blazars), and the most likely origin of the MGF candidates is simply statistical flukes induced by the Poisson statistics of a steady gamma-ray flux. Indeed, we confirmed that the number expected by Poisson statistics and point source fluxes is in rough agreement with that of the detected MGF candidates.

There is an excess of the MGF distribution in Fig. 2 at $\theta \gtrsim$ $2.0^{\circ}$ compared with PSF, which implies a contribution of MGFs that are not caused by point sources. We will see that these are likely caused by the diffuse gamma-ray background (see below). To remove MGF candidates related to bright point sources, MGF candidates are excluded from the final sample if their nearest 3FGL source flux is larger than $4 \times 10^{-9}$ photons $/ \mathrm{cm}^{2} / \mathrm{s}$ and angular separation is smaller than $2.0^{\circ}$. The number of 3FGL sources exceeding this flux threshold is 293 . The result of this cut is shown in Table 1 as "2nd cut". 
Table 1. The number of detected MGF candidates $\left(N_{\mathrm{obs}}^{2 \mathrm{ph}}\right.$ and $N_{\mathrm{obs}}^{3 \mathrm{ph}}$ for $N_{\mathrm{ph}}=2$ and 3, respectively). "No cut" is the result of our blind search for MGFs without any event cut. The "1st cut" is to remove MGF candidates associated with GRB 090519. The "2nd cut" is the result after removing MGF events that are caused by nearby bright sources [brighter than a photon flux of $4 \times 10^{-9}$ photons $\mathrm{cm}^{-2} \mathrm{~s}^{-1}(1 \mathrm{GeV}<E<100 \mathrm{GeV})$ with angular separation less than $2.0^{\circ}$ ]. The "3rd cut" is removing regions close to the Galactic disk $\left(|b|<20^{\circ}\right)$. The expected numbers of false events caused by the Poisson statistics of the diffuse gamma-ray background flux are shown as $N_{\text {pred }}^{2 \mathrm{ph}}$ in the 2 nd and $3 \mathrm{rd}$ cut results.

\begin{tabular}{|c|c|c|c|c|c|c|c|c|}
\hline \multirow[t]{2}{*}{$\begin{array}{l}\text { Cuts } \\
f_{\text {sky }}{ }^{\text {a }}\end{array}$} & \multicolumn{2}{|c|}{$\begin{array}{c}\text { No cut } \\
1.0\end{array}$} & \multicolumn{2}{|c|}{$\frac{1 \mathrm{st} \text { cut }}{1.0}$} & \multicolumn{2}{|c|}{$\frac{2 \text { nd cut }}{0.96}$} & \multicolumn{2}{|l|}{$\begin{array}{c}\text { 3rd cut } \\
0.68\end{array}$} \\
\hline & $N_{\mathrm{obs}}^{2 \mathrm{ph}}$ & $N_{\mathrm{obs}}^{3 \mathrm{ph}}$ & $N_{\mathrm{obs}}^{2 \mathrm{ph}}$ & $N_{\mathrm{obs}}^{3 \mathrm{ph}}$ & $N_{\text {obs }}^{2 \mathrm{ph}}\left(N_{\text {pred }}^{2 \mathrm{ph}}\right)$ & $N_{\mathrm{obs}}^{3 \mathrm{ph}}$ & $N_{\text {obs }}^{2 \mathrm{ph}}\left(N_{\text {pred }}^{2 \mathrm{ph}}\right)$ & $N_{\mathrm{obs}}^{3 \mathrm{ph}}$ \\
\hline$\Delta t=1 \mathrm{~ms}$ & 17 & 0 & 14 & 0 & $1(2.7)$ & 0 & $0\left(1.2 \times 10^{-1}\right)$ & 0 \\
\hline$\Delta t=2 \mathrm{~ms}$ & 33 & 0 & 29 & 0 & $4(5.3)$ & 0 & $0\left(2.3 \times 10^{-1}\right)$ & 0 \\
\hline$\Delta t=5 \mathrm{~ms}$ & 68 & 1 & 62 & 0 & $17(13)$ & 0 & $0\left(5.8 \times 10^{-1}\right)$ & 0 \\
\hline$\Delta t=10 \mathrm{~ms}$ & 133 & 1 & 127 & 0 & 38 (27) & 0 & $0(1.0)$ & 0 \\
\hline
\end{tabular}

a The remaining sky fraction after each event cut.

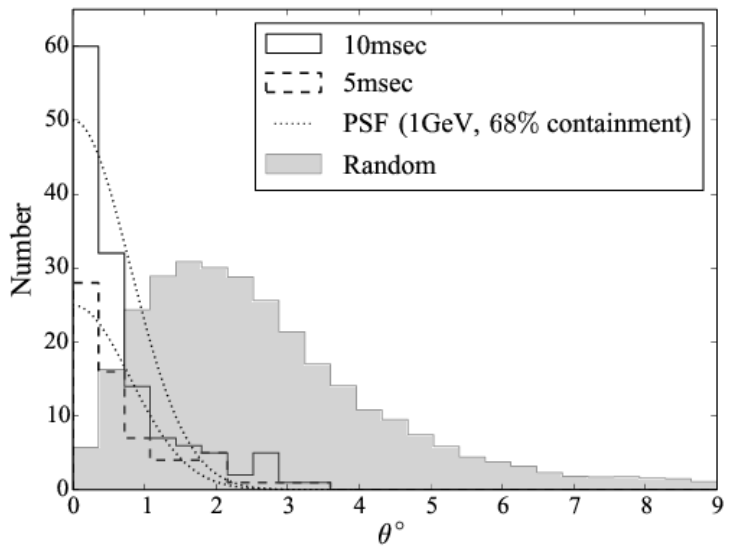

Figure 2. Angular separation distribution from each MGF candidate $\left(N_{\mathrm{ph}}=2\right)$ to the nearest 3FGL catalog source, for $\Delta t=5$ and $10 \mathrm{~ms}$. The Fermi-LAT point spread function profile (at $1 \mathrm{GeV}, 68 \%$ containment) is also shown by the dashed curves (two same profiles scaled to the histograms of two $\Delta t$ values). For comparison, the expected distribution when MGFs are randomly distributed on the sky is also shown.

\subsection{False MGFs by the Diffuse Background}

Even after removing the regions affected by bright point sources, MGF candidates remain. These may be statistical flukes caused by Poisson statistics of constant gamma-ray flux of the diffuse gammaray background. Here we estimate the number of MGF candidates expected by this. For this purpose, we firstly divide all sky into $72 \times 36$ regular square pixels in $\mathrm{x}$ - and $\mathrm{y}$-axis directions on the Aitoff projection (i.e., a pixel scale of $\sim 5^{\circ}$ ), and the spatial direction and surface area of the $j$-th pixel are denoted by solid angle $\Omega_{j}$ and $\Delta \Omega_{j}$. We then create a count map and an exposure map of gamma-rays selected by the criteria described in Section 2 using the Fermi analysis tool of gtltcube and gtexpcube. By dividing the count map (in photons) by the exposure map (in $\mathrm{cm}^{2} \mathrm{~s}$ ) and the surface area $\Delta \Omega_{j}$, we get an intensity map (in photons $\mathrm{cm}^{-2} \mathrm{~s}^{-1} \mathrm{sr}^{-1}$ ) of the diffuse gamma-ray background, $J\left(\Omega_{j}\right)$.

Next, we divide the whole mission time into time bins of $\Delta T=10 \mathrm{~min}$, and the $i$-th time bin is denoted as $T_{i}$. The exposure map $\varepsilon\left(T_{i}, \Omega_{j}\right)$ for each time bin is calculated with the same angular pixel binning on the sky. We need to calculate the effective area of the detector in each time bin to estimate the expected number of Poisson-induced MGF events. Fermi typically surveys $2 \pi$ sr in $1.5 \mathrm{hr}$, and change of effective area for a given pixel in $10 \mathrm{~min}$ is not large and hence we approximate it to be constant in each time bin. It should be noted that the effective area is relatively flat in the gamma-ray energy range adopted in this work, and we assume that it is constant against gamma-ray energy. We calculate the exposure time $T_{\exp }\left(T_{i}\right)$ in each time bin by adding all the GTIs. Then we can estimate $A_{\text {eff }}\left(T_{i}, \Omega_{j}\right)$ from exposure $\varepsilon\left(T_{i}, \Omega_{j}\right)$ calculated by the Fermi Science Tool, from the relation

$$
\varepsilon\left(T_{i}, \Omega_{j}\right)=A_{\text {eff }}\left(T_{i}, \Omega_{j}\right) T_{\exp }\left(T_{i}\right),
$$

where $A_{\text {eff }}$ is the effective area averaged about energy and angular resolutions.

Then the expected mean photon count within the MGF search time window $\Delta t$ and the search radius ( $2 \mathrm{deg}$ ) is

$$
\lambda\left(T_{i}, \Omega_{j}\right)=J\left(\Omega_{j}\right) A_{\mathrm{eff}}\left(T_{i}, \Omega_{j}\right) \omega \Delta t,
$$

where $\omega$ is the solid angle of the search circle. In the Poisson statistics, the probability that $N_{\text {ph }}$ photons arrive is

$$
P\left(T_{i}, \Omega_{j} ; N_{\mathrm{ph}}, \Delta t\right)=\frac{\lambda\left(T_{i}, \Omega_{j}\right)^{N_{\mathrm{ph}}}}{N_{\mathrm{ph}} !} \exp \left[-\lambda\left(T_{i}, \Omega_{j}\right)\right] .
$$

The expected number of MGFs in a time bin $T_{i}$ at a pixel $\Omega_{j}$ is then obtained by multiplying the number of trials, i.e., the number of time windows, $T_{\exp }\left(T_{i}\right) / \Delta t$, and the number of search circles, $\Delta \Omega_{j} / \omega$ :

$$
N\left(T_{i}, \Omega_{j} ; N_{\mathrm{ph}}, \Delta t\right)=P\left(T_{i}, \Omega_{j} ; N_{\mathrm{ph}}, \Delta t\right) \frac{T_{\exp }\left(T_{i}\right)}{\Delta t} \frac{\Delta \Omega_{j}}{\omega} .
$$

Then the total expected number of Poisson-induced MGF events can be calculated by summing up $N\left(T_{i}, \Omega_{j} ; N_{\mathrm{ph}}, \Delta t\right)$ for $T_{i}$ and $\Omega_{j}$. Note that the event cuts introduced in the previous section can be taken into account by removing regions around bright point sources from $\Delta \Omega_{j}$.

The expected event numbers of false MGFs calculated in this way are compared with the observed numbers after the 2 nd cut in Table 1, and also in Fig. 3 as a histogram along the Galactic latitude. The agreement between the expected and observed numbers is good, and hence most of the observed $N_{\mathrm{ph}}=2$ events after the 2 nd cut are considered to be produced by Poisson statistics of the diffuse background. It should be noted that all these events are found at low latitude regions of $|b|<20^{\circ}$. Therefore we make the 


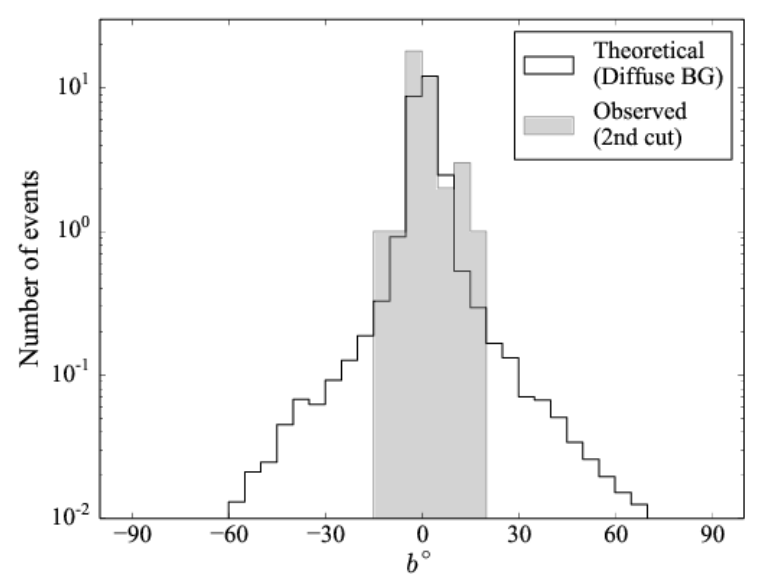

Figure 3. The Galactic latitude distribution of the MGF candidates with $N_{\mathrm{ph}}=2$ and $\Delta t=10 \mathrm{~ms}$ after the 2 nd cut (gray histogram). The expected distribution from the Poisson statistics of the diffuse gamma-ray background is shown as solid histogram.

final 3rd cut to remove events of $|b|<20^{\circ}$. Then the final result is that we found no MGF candidates after the three event cuts, and this will be used to set a limit on the gamma-ray/radio flux ratio of FRBs in section 4.

\section{IMPLICATIONS FOR FRBS}

\subsection{Modeling the population of FRBs}

To set limits on physical quantities of FRBs by the no detection of MGFs, we first model the luminosity and rate evolution of FRBs. The luminosity function of FRBs is totally unknown, and here we simply use the standard candle approximation. Based on the typical peak flux $F_{\nu}=0.5 \mathrm{Jy}$ of FRBs at observing frequency of $\nu=$ $1.4 \mathrm{GHz}$, and taking $z \sim 0.5$ as FRB redshifts (Keane \& Petroff 2015), we set the radio luminosity of FRBs to be $\left(\nu L_{\nu}\right)_{r}=1.1 \times$ $10^{43} \mathrm{erg} \mathrm{s}^{-1}$ at the rest-frame reference frequency $\nu_{r}=2.1 \mathrm{GHz}$.

To relate gamma-ray flux and radio flux of FRBs, we define gamma-ray to radio luminosity ratio in the rest-frame of a FRB as

$$
\xi \equiv \frac{\left(\nu L_{\nu}\right)_{\gamma}}{\left(\nu L_{\nu}\right)_{r}}
$$

where gamma-ray luminosity is at a gamma-ray energy of $1.5 \mathrm{GeV}$ (corresponding to the minimum Fermi gamma-ray energy $1 \mathrm{GeV}$ adopted in this work for $z=0.5$ ), and radio luminosity at $\nu=\nu_{r}$. We assume that $\xi$ is the same for all FRBs, and that FRBs have a power-law gamma-ray spectrum with a photon spectral index $\Gamma$, i.e., $d L_{\gamma} / d \epsilon \propto \epsilon^{-\Gamma}$, where $d L_{\gamma} / d \epsilon$ is the differential gamma-ray photon luminosity per unit gamma-ray energy at the rest-frame. Then we can calculate the gamma-ray flux $d F_{\gamma} / d \epsilon_{\text {obs }}$ as a function of observed gamma-ray energy $\epsilon_{\mathrm{obs}}$ for a FRB at a redshift $z$ if $\xi$ and $\Gamma$ are specified. Since there is no knowledge of $\Gamma$ observationally, we assume $\Gamma=2.0$. If we change $\Gamma$ by \pm 2 , the final constraint on $\xi$ changes only by about $2 \%$.

We model the comoving FRB rate density and its evolution as $\Phi_{\mathrm{FRB}}(z, \beta)=\Phi_{0}(1+z)^{\beta}$, and the rate in all sky for FRBs in a redshift range $z_{1}<z<z_{2}$ is

$$
\mathcal{R}_{\mathrm{FRB}}\left(z_{1}, z_{2} ; \beta\right)=\int_{z_{1}}^{z_{2}} d z \frac{\Phi_{\mathrm{FRB}}}{1+z} \frac{d V}{d z},
$$

where $d V / d z$ is the comoving volume element per unit redshift for all sky, and $(1+z)^{-1}$ is the cosmological time dilation factor. For each assumed value of $\beta$, we determine $\Phi_{0}$ by the fluencecomplete rate of $\mathcal{R}_{\mathrm{FRB}}(0.19<z<0.89) \sim 2.5_{-1.6}^{+3.2} \times 10^{3}$ day $^{-1}$ based on 9 FRBs ( 8 by Parkes, 1 by Arecibo) at $0.19<z<0.89$ with fluence above $2 \mathrm{Jy}$ ms (Keane \& Petroff 2015). The value of $\Phi_{0}$ changes by a factor of less than 1.5 if we use another similar rate estimate of $\mathcal{R}_{\mathrm{FRB}}(0.35<z<1.3) \sim 6_{-3}^{+4} \times 10^{3}$ day $^{-1}$ (for 9 Parkes FRBs, not fluence-complete down to $0.9 \mathrm{Jy} \mathrm{ms}$ ) by Champion et al. (2015). We will present results for a range of $\beta$ $=0-4$. If FRBs are produced by short life-time phenomena from star formation (e.g., core collapse supernovae or young magnetars), their rate should trace the star formation history in the universe ( $\beta \sim 3-4$ to $z \sim 1$ ), while a smaller value $(\beta \sim 2)$ is expected if FRBs are mergers of binary neutron stars or white dwarfs (Totani 1999; Totani et al. 2008; Dominik et al. 2013; Madau \& Dickinson 2014).

\subsection{Upper-limit on gamma-ray / radio luminosity ratio}

Now we can calculate the expected number of MGFs by FRBs as a function of $\xi$, thus deriving the upper bound on this parameter by the observational result of no MGF detection for $N_{\mathrm{ph}}=2 \mathrm{MGF}$ events after the 3 rd event cut. We have searched MGF events with four values of $\Delta t$, but here we use $\Delta t=3 \mathrm{msec}$ to match the typical observed duration of FRBs. In a similar way to the calculation in section 3.2, we again calculate for each pixel of the sky to a direction $\Omega_{j}$ in each 10-min bin $T_{i}$ of observing time. The expected number of detectable gamma-rays from a FRB to a direction $\Omega_{j}$ at redshift $z$ in a MGF search time interval $\Delta t$ is given as

$$
N_{\gamma}=\Delta t \int_{1 \mathrm{GeV}}^{100 \mathrm{GeV}} d \epsilon_{\mathrm{obs}} A_{\mathrm{eff}}\left(T_{i}, \Omega_{j}\right) \frac{d F_{\gamma}\left(\epsilon_{\mathrm{obs}} ; z, \xi\right)}{d \epsilon_{\mathrm{obs}}} .
$$

For a search of MGFs of $N_{\mathrm{ph}}$ photons, we can define the maximum redshift $z_{\max }\left(\xi, T_{i}, \Omega_{j}\right)$ within which a FRB should be detected as a MGF event, by equating $N_{\gamma}=N_{\mathrm{ph}}$. Then the expected number of all MGF events caused by FRBs can be calculated as

$$
N_{\mathrm{MGF}}\left(T_{i}, \Omega_{j}\right)=\mathcal{R}_{\mathrm{FRB}}\left[0, z_{\max } ; \beta\right] \frac{\Delta \Omega_{j}}{4 \pi} T_{\exp }\left(T_{i}\right),
$$

and the final total expected number of MGFs in all sky and the observing period, $N_{\mathrm{MGF}}^{\text {tot }}$, is obtained by summing up $N_{\mathrm{MGF}}\left(T_{i}, \Omega_{j}\right)$ for $T_{i}$ and $\Omega_{j}$. The event cuts about the Fermi point source regions and Galactic latitude can be taken into account in $\Delta \Omega_{j}$.

The expected number $N_{\text {MGF }}^{\text {tot }}$ increases with increasing $\xi$ because FRBs can be detected to larger distances. Then no MGF detection after the 3rd event cut sets an upper limit on $\xi$, which is estimated by equating $N_{\mathrm{MGF}}^{\mathrm{tot}}(\xi)=3.09$, corresponding to $95 \%$ upper limit on the expected value of the Poisson statistics. The results are summarized in Table 2, and the upper limits are found to be $\xi=(4.2-12) \times 10^{7}$ for $0 \leqslant \beta \leqslant 4$. The maximum redshift $z_{\max }$ depends on the direction to a FRB with respect to the Fermi-LAT FoV center, and it is 0.02 for $\xi \sim 10^{8}$ when a FRB is located at the FoV center. This is much smaller than that of FRBs detected in radio bands ( $z \gtrsim 0.5$ ), confirming that Fermi-LAT is sensitive to FRBs at smaller distances than those detected in radio bands. Correspondingly, our limit on $\xi$ is stronger than that obtained from non-detection of radio-detected FRBs by Fermi-LAT (typically $\xi \lesssim 4 \times 10^{10}$ ). For comparison, Tendulkar et al. (2016) investigated Fermi-GBM, Swift-BAT and Konus-WIND data at detected 16 FRB fields, setting an upper limit on soft gamma-ray to radio flux ratio of $\xi_{\text {soft } \gamma} \lesssim 10^{8}-10^{10}$. 
Table 2. The $2 \sigma$ upper limits on gamma-ray to radio flux ratio $(\xi)$ for $\beta=$ $0-4$. The normalization of the volumetric FRB rate at $z=0\left(\Phi_{0}\right)$ is also shown. FRB duration is assumed to be $\Delta t=3 \mathrm{msec}$.

\begin{tabular}{ccc}
\hline \hline$\beta$ & $\begin{array}{c}\Phi_{0} \\
{\left[\mathrm{Gpc}^{-3} \mathrm{yr}^{-1}\right]}\end{array}$ & {$\left[\equiv\left(\nu L_{\nu}\right)_{\gamma} /\left(\nu L_{\nu}\right)_{r}\right]$} \\
\hline 0 & $1.2_{-0.8}^{+1.5} \times 10^{4}$ & $<4.2_{-1.7}^{+2.4} \times 10^{7}$ \\
1 & $7.4_{-4.4}^{+9.4} \times 10^{3}$ & $<5.2_{-2.0}^{+4.3} \times 10^{7}$ \\
2 & $4.5_{-2.9}^{+5.7} \times 10^{3}$ & $<7.0_{-2.8}^{+5.0} \times 10^{7}$ \\
3 & $2.7_{-1.7}^{+3.5} \times 10^{3}$ & $<9.2_{-3.8}^{+7.8} \times 10^{7}$ \\
4 & $1.6_{-1.0}^{+2.1} \times 10^{3}$ & $<1.2_{-0.4}^{+1.2} \times 10^{8}$ \\
\hline
\end{tabular}

\subsection{Discussion}

The extremely high brightness temperature of FRBs implies that their radio emission is likely coherent radiation, and it is reasonable to expect that it is similar to that of radio pulsars. The gammaray to radio ratio is $\xi=10^{4}-10^{8}$ for gamma-ray pulsars in the Second Fermi Large Area Telescope Catalog of Gamma-Ray Pulsars (2PC; Abdo et al. 2013). Some radio pulsars exhibit radio efficiencies of $\sim 10^{-2}-10^{-4}$ with respect to spin-down luminosity (Szary et al. 2014), indicating that $\xi$ should be smaller than $10^{4}$ assuming that gamma-ray luminosity cannot exceed the spin-down luminosity. Therefore $\xi$ of pulsars are distributed in a wide range, and our upper limit of $\xi \lesssim 10^{8}$ does not exclude a possibility that FRBs have a similar GeV gamma-ray to radio flux ratio to pulsars. Recently Repeating bursts were discovered from FRB 121102 (Spitler et al. 2014; Spitler et al. 2016; Scholz et al. 2016), and the top candidate progenitor model would be super-giant pulses from pulsars. This is also consistent with our results if the gamma-ray to radio flux ratio of giant pulses are similar to that of normal pulsar emission.

Giant flares from soft gamma-ray repeaters (magnetars) are also discussed as a popular hypothesis for the FRB origin (Popov \& Postnov 2010; Thornton et al. 2013; Lyubarsky 2014; Kulkarni et al. 2014). Tendulkar et al. (2016) placed an upper limit of $<1.1 \mathrm{MJy}$ on the radio flux by the non-detection of a radio single pulse event from the giant flare event of SGR 1806-20 on December 27, 2004, and it is interesting to compare our limit to that of this giant magnetar flare. Unfortunately there is no direct $\mathrm{GeV}$ observation of the SGR 1806-20 giant flare in 2004, but we can estimate a reasonable flux level as follows. Frederiks et al. 2007 reported $20 \mathrm{keV}-10 \mathrm{MeV}$ spectrum of the giant flare using the Compton reflection from the Moon. Though non-thermal emission was not detected for the initial flare, a power-law component extending to $10 \mathrm{MeV}$ with a photon index $\Gamma=1.7$ was detected in the pulsating tail phase. The energy flux of the non-thermal component in $1-10 \mathrm{MeV}$ is $1.9 \%$ of the thermal component in $20-300 \mathrm{keV}$. If we assume the same percentage for the initial flare, the non-thermal flux of the initial flare is estimated to be $0.21 \mathrm{erg} \mathrm{cm}^{-2} \mathrm{~s}^{-1}$, which is much lower than the thermal flux at $<\mathrm{MeV}$ and hence consistent with no detection. Assuming that the power-law spectrum is extending to $\mathrm{GeV}$ with the same photon index, we estimate $\nu F_{\nu}$ flux at $1 \mathrm{GeV}$ to be $0.51 \mathrm{erg} \mathrm{cm}^{-2} \mathrm{~s}^{-1}$, and combined with the radio upper limit, we obtain a lower limit to the $\mathrm{GeV}$ to radio flux ratio as $\xi \gtrsim 10^{7.5}$. This is marginally consistent with the upper limit on $\xi$ derived by our work, and hence our result does not exclude a possibility that FRBs have a similar $\mathrm{GeV}$ to radio flux ratio to magnetar giant flares.

Binary neutron star mergers are also proposed as a possible candidate for catastrophic FRB events, and in this case we may expect associations between FRBs and short gamma-ray bursts (sGRBs) (Totani 2013; Zhang 2014). Only one short GRB 090510 was detected in our MGF search in $\mathrm{GeV}$, and note that sGRBs are efficiently detected in soft gamma-rays with a trigger time scale much longer than the search made here. This means that our search is not optimized to constraining sGRBs. Furthremore, the upper limits on $\xi$ derived in this work is valid only for sources whose event rate is similar to that of FRBs, but it is known that the event rate of sGRBs ( $\sim 10 \mathrm{yr}^{-1} \mathrm{Gpc}^{-3}$; Coward et al. 2012) is much smaller than that of FRBs $\left(\sim 10^{4} \mathrm{yr}^{-1} \mathrm{Gpc}^{-3}\right)$. Therefore our results do not strongly constrain the possible sGRB-FRB connection, and it is possible that a small fraction of FRBs are observed as sGRBs.

\section{CONCLUSION}

In this paper we searched millisecond-duration gamma-ray flashes (MGFs) in the 7-year Fermi-LAT data, motivated by the possible gamma-ray counterpart to FRBs. Since Fermi-LAT is observing wider field-of-view compared with radio telescopes finding FRBs, FRBs at shorter distances are expected to be detected in the FermiLAT, which are out of search fields of view of radio telescopes. Therefore we performed a blind search of multiple gamma-ray events within a circle of point spread function and a time interval $\Delta t=1-10 \mathrm{msec}$, and found about $100 \mathrm{MGF}$ candidates. There is only one event detected with three photons or more, and it is related to a bright short-duration gamma-ray burst GRB 090519. Examination of other events indicates that all of them can be explained by the Poisson statistics of steady gamma-ray flux from bright point sources or diffuse gamma-ray background. After removing regions of bright point sources, there is no MGF event in high Galactic latitude regions of $|b|>20^{\circ}$.

We then used this result to place an upper limit on the gammaray to radio flux ratio of FRBs, $\xi \equiv\left(\nu L_{\nu}\right)_{\gamma} /\left(\nu L_{\nu}\right)_{r}$. In the calculation, we need to assume the comoving rate density and its evolution of FRBs, and assuming a power-law type evolution, $\Phi_{\mathrm{FRB}}=\Phi_{0}(1+z)^{\beta}$, we derived $\xi<(4.2-12) \times 10^{7}$ for $\beta=1-4$. This limit is stronger than those obtained by no gamma-ray detection of known FRBs by about three orders of magnitude. The limit is comparable with the largest values found for pulsars. However, $\xi$ of pulsars is distributed widely in many orders of magnitudes, and we cannot exclude that FRBs have similar values of $\xi$ to pulsars in general. We also compared our limit with the spectral energy distribution of the 2004 giant flare of SGR 1806-20. The upper bound on the radio flux for this flare is marginally consistent with our limit of $\xi$, if a non-thermal power-law component up to $\mathrm{GeV}$ exists in the initial flare phase and its energy fraction with respect to the thermal emission is similar to that of pulsating tail phase emission.

\section{ACKNOWLEDGEMENTS}

We thank the anonymous Referee for the helpful remarks and suggestions. This work was supported by JSPS KAKENHI Grant Numbers 15K05018 and 40197778.

\section{REFERENCES}

Abdo A. A., et al., 2013, ApJS, 208, 17

Acero F., et al., 2015, ApJS, 218, 23 


\section{Yamasaki, Totani, \& Kawanaka}

Ackermann M., et al., 2010, ApJ, 716, 1178

Atwood W. B., et al., 2009, ApJ, 697, 1071

Burke-Spolaor S., Bannister K. W., 2014, ApJ, 792, 19

Champion D. J., et al., 2015, preprint, (arXiv:1511.07746)

Connor L., Sievers J., Pen U.-L., 2015, preprint, (arXiv: 1505 . 05535)

Cordes J. M., Wasserman I., 2015, preprint, (arXiv:1501.00753)

Coward D. M., et al., 2012, Monthly Notices of the Royal Astronomical Society, 425, 2668

Dominik M., Belczynski K., Fryer C., Holz D. E., Berti E., Bulik T., Mandel I., O'Shaughnessy R., 2013, ApJ, 779, 72

Falcke H., Rezzolla L., 2014, A\&A, 562, A137

Frederiks D. D., Golenetskii S. V., Palshin V. D., Aptekar R. L., Ilyinskii V. N., Oleinik F. P., Mazets E. P., Cline T. L., 2007, Astronomy Letters, 33,1

Kashiyama K., Ioka K., Mészáros P., 2013, ApJ, 776, L39

Keane E. F., Petroff E., 2015, MNRAS, 447, 2852

Keane E. F., Stappers B. W., Kramer M., Lyne A. G., 2012, MNRAS, 425, L71

Keane E. F., et al., 2016, Nature, 530, 453

Kulkarni S. R., Ofek E. O., Neill J. D., Zheng Z., Juric M., 2014, ApJ, 797, 70

Lorimer D. R., Bailes M., McLaughlin M. A., Narkevic D. J., Crawford F., 2007, Science, 318, 777

Lyubarsky Y., 2014, MNRAS, 442, L9

Madau P., Dickinson M., 2014, ARA\&A, 52, 415

Masui K., et al., 2015, Nature, 528, 523

Mingarelli C. M. F., Levin J., Lazio T. J. W., 2015, ApJ, 814, L20

Petroff E., et al., 2015, MNRAS, 454, 457

Planck Collaboration et al., 2015, preprint, (arXiv:1502.01589)

Popov S., Postnov K., 2010, in Evolution of Cosmic Objects through their Physical Activity. pp 129-132

Ravi V., Shannon R. M., Jameson A., 2015, ApJ, 799, L5

Scholz P., et al., 2016, preprint, (arXiv: 1603.08880)

Spitler L. G., et al., 2014, ApJ, 790, 101

Spitler L. G., et al., 2016, Nature, 531, 202

Szary A., Zhang B., Melikidze G. I., Gil J., Xu R.-X., 2014, ApJ, 784, 59

Tendulkar S. P., Kaspi V. M., Patel C., 2016, preprint, (arXiv: 1602.02188)

Thornton D., et al., 2013, Science, 341, 53

Totani T., 1999, The Astrophysical Journal, 511, 41

Totani T., 2013, PASJ, 65

Totani T., Morokuma T., Oda T., Doi M., Yasuda N., 2008, PASJ, 60, 1327

Zhang B., 2014, ApJ, 780, L21 
This paper has been typeset from a $\mathrm{T}_{\mathrm{E}} \mathrm{X} / \mathrm{LAT}_{\mathrm{E}} \mathrm{X}$ file prepared by the author. 